\title{
METHODS AND MODELS FOR TEXTURE ANALYSIS OF LUNG PATHOLOGICAL CHANGES BASED ON COMPUTED TOMOGRAPHY FOR COVID-19 DIAGNOSIS
}

\author{
A.S. Kents ${ }^{1, *}$, Yu.A. Hamad ${ }^{2}$, K.V. Simonov ${ }^{3}$, A.G. Zotin ${ }^{4}$ \\ ${ }^{1}$ Federal State-Financed Institution Federal Siberian Research Clinical Centre, Federal Medical Biological Agency, \\ 26 Kolomenskaya st., 660037, Krasnoyarsk, Russian Federation - lika.kents@ mail.ru \\ ${ }^{2}$ Institute of Space and Information Technologies, Siberian Federal University, 79 Svobodny st., Krasnoyarsk 660041, Russian \\ Federation-y.albayati8@gmail.com \\ ${ }^{3}$ Institute of Computational Modelling of the SB RAS, 50/44 Akademgorodok, Krasnoyarsk 660036, Russian Federation - \\ simonovkv@icm.krasn.ru \\ ${ }^{4}$ Institute of Computer Science and Telecommunications, Reshetnev Siberian State University of Science and Technology, \\ 31 Krasnoyarsky Rabochy ave., Krasnoyarsk, 660037. Russian Federation - zotin@ sibsau.ru
}

\author{
Commission V, WG II/10
}

KEY WORDS: CT image, Lung Pathology from COVID-19, Pulmonary Fibrosis, Prognosis of outcomes, Texture Image Analysis, Color-coded Contrasting

\begin{abstract}
:
In recent years computed tomography of the lungs has been the most common diagnostic procedure aimed at detection of the pathological changes associated with COVID-19. The study is aimed at the use of the developed algorithmic support in combination with texture (geometric) analysis to highlight a number of indicators characterizing the clinical state of the object of interest. Processing is aimed at the solution of a number of diagnostic tasks such as highlighting and contrasting the objects of interest, taking into account the color coding. Further, an assessment is performed according to the appropriate criteria in order to find out the nature of the changes and increase both the visualization of pathological changes and the accuracy of the X-ray diagnostic report. For these purposes, it is proposed to use preprocessing algorithms for a series of images in dynamics. Segmentation of the lungs and areas of possible pathology are performed using wavelet transform and Otsu threshold value. Delta-maps and maps obtained using Shearlet transform with contrasting color coding are used as a means of visualization and selection of features (markers). The analysis of the experimental and clinical material carried out in the work shows the effectiveness of the proposed combination of methods for studying of the variability of the internal geometric features (markers) of the object of interest in the images.
\end{abstract}

\section{INTRODUCTION}

Currently there is a great threat to humanity due to the spread of the infection caused by the novel coronavirus (Radiation diagnosis of coronavirus disease (COVID-19): organization, methodology, interpretation of results, 2020). High mortality is associated with acute severe respiratory failure resulting in hospitalization and intubation. These circumstances are the basis for taking urgent measures and constant monitoring of patients. But after recovery and discharge more distant consequences are possible, associated with the possible preservation of residual changes and the formation of pulmonary fibrosis.

The observed changes lead to a decrease in the working surface of the lungs therefore they can lead to a decrease in the quality of life and the addition of others, in particular, bacterial infections due to a decrease in ventilation of the affected sections. In this regard early diagnosis and dynamic observation of patients with the use of radiation diagnostic methods helps to prevent possible consequences. This is due to the adjustment and greater effectiveness of therapy. And also in the correlation of the obtained CT images with the patient's condition.

The radiation method of chest diagnostics is used on an outpatient and inpatient basis. It is the main instrumental method for identification of the suspected coronavirus infection. Radiography is technically the simplest and most accessible method for preliminary diagnosis of the disease. At the same time it is not the most informative method in relation to CT diagnosis.

When analyzing a medical image, high quality is required which is a combination of several factors. It also requires the professionalism and experience of a specialist radiologist. Note that the datasets associated with $\mathrm{X}$-ray imaging continue to grow at a rapid pace. At the same time the number of specialist radiologists is limited. These circumstances contribute to a dramatic increase in the workload of radiologists. Research shows that in some cases the radiologist interprets the image every 3-4 seconds over an 8-hour workday (McDonald R.J. et al., 2015).

\footnotetext{
* Corresponding author
} 
To improve the quality and speed of image analysis various additional visualization techniques are proposed. These methods can affect the above criteria and the final conclusion of the radiologist. To obtain the final result the image undergoes a series of transformations. In this case the main method is the segmentation method which is widely used when working with the chest $\mathrm{x}$-ray.

The paper proposes the following: a method of interpretation, including models and methods of texture analysis of pathological changes in the lungs on CT images with COVID19. The method is intended to improve the visualization of these areas to improve the accuracy of diagnosis and expand the radiological conclusion. It is proposed to study the textural feature in the form of "frosted glass" manifestation on low-dose CT images. This allows you to reduce radiation exposure to the patient without losing image quality.

Within the framework of the proposed interpretation method, the following interrelated tasks are solved:

- search and determination of a number of texture markers on the CT images;

- allocation of the border of the involved parenchyma with quantitative characteristics for each lung in percentage terms;

- correlation of changes in the lung and the stage of the disease with the differentiation of changes in the density component to control the dynamics of denser zones and form a prognosis;

- implementation of dynamic observation of patients in the delayed period;

- development of an atlas of reference images for early detection and prognosis of the development of fibrosis.

\section{RELATED WORKS}

At the present time in this period of the pandemic a set of retrospective data is starting in different areas of research. In our work the study is performed using CT images of the lungs. The most indicative sign is the determination of the severity of the lung damage.

In the work of Chinese scientists a study was conducted on the basis of 73 cases and distributions of indicators were constructed (Liu KC et al., 2020). Also Bernheim et al. (Bernheim A et al., 2020) an analysis of the results of computed tomography of 121 symptomatic patients, COVID-19 infection, was performed depending on the time between the onset of symptoms and the initial detection on a computed tomogram. A correlation has been shown between the CT symptoms and the stage of the disease. The authors' research is essential for the recognition and visualization of pathology depending on the time of infection.

However, many severely ill patients often have the disease. A study by the Chinese scientists (Liu KC et al., 2020) analyzes the characteristics of a CT scan of the chest with COVID-19 based on the severity of the disease. This reflects the relationship between CT characteristics and disease severity more accurately compared to the studies mentioned above. This article also performs a dynamic CT scan of the lungs in different groups to highlight delayed changes or regression.

In addition to the usual image processing, specialists use various methods of computer vision and machine learning. The study by Huseyin Yasar et al. analyzes a total of 1,396 CT images of the lungs (386 COVID-19 and 1,010 Non-COVID19), which were automatically classified. This study used a Convolutional Neural Network (CNN). It is one of the deep learning methods that offer automatic classification of the CT images of the lungs for early diagnosis of COVID-19 disease. In addition, k-Nearest Neighbors (k-NN) and support vector machines (SVM) were used to compare the classification success of deep learning with machine learning. As part of this study a 23-level CNN architecture was developed and used as a classifier. The highest values of the mean sensitivity, specificity, accuracy, F-1 and AUC estimates obtained as a result of the study were $0.9197,0.9891,0.9473,0.9058,0.9888$ which is high (Huseyin Yasar et al., 2021).

With regard to the study by Shen et al. (Shen S. et al., 2020), the lesion levels on the CT images of lungs belonging to 44 patients with COVID-19 were noted by radiologists and computers, respectively. The study results show that computerized labeling is a reliable alternative method for determination of the severity and spread of COVID-19 pneumonia.

The review study by Long and Ehrenfeld (Long JB et al., 2020) emphasizes that the use of artificial intelligence techniques to mitigate the impact of the COVID-19 outbreak crisis is an important requirement. In this context some applications have been implemented regarding the automatic classification of Covid-19 and Non-COVID-19 CT images using artificial intelligence techniques.

There are also works in which computer vision methods and segmentation algorithms are used, which are performed using the method of principal component analysis (PCA) and discrete wavelet transform (DWT). Based on the results of the primary segmentation, binarization is performed based on the Otsu threshold determination method. Next, the formation of the contour representation is carried out using the Shearlet transform and color coding. Then, the characteristics of the texture of objects of interest are calculated (Kents A. et al., 2020).

In the course of the study, it was revealed that the accuracy of a specialist's assessment does not differ much from the accuracy of an assessment with computer processing. However, this depends on the qualifications of the specialist. To highlight the features of images, it is necessary to consider a larger list of criteria and variability of indicators. Moreover, a specialist cannot evaluate a number of criteria without using software.

The visualization method proposed in our work, accompanied by texture analysis, makes it possible to obtain a number of useful indicators (markers). These indicators provide an objective and quantitative assessment of the heterogeneity of the pathology by analyzing the differences and patterns in the set of CT images (Zotin A. et al., 2020). 


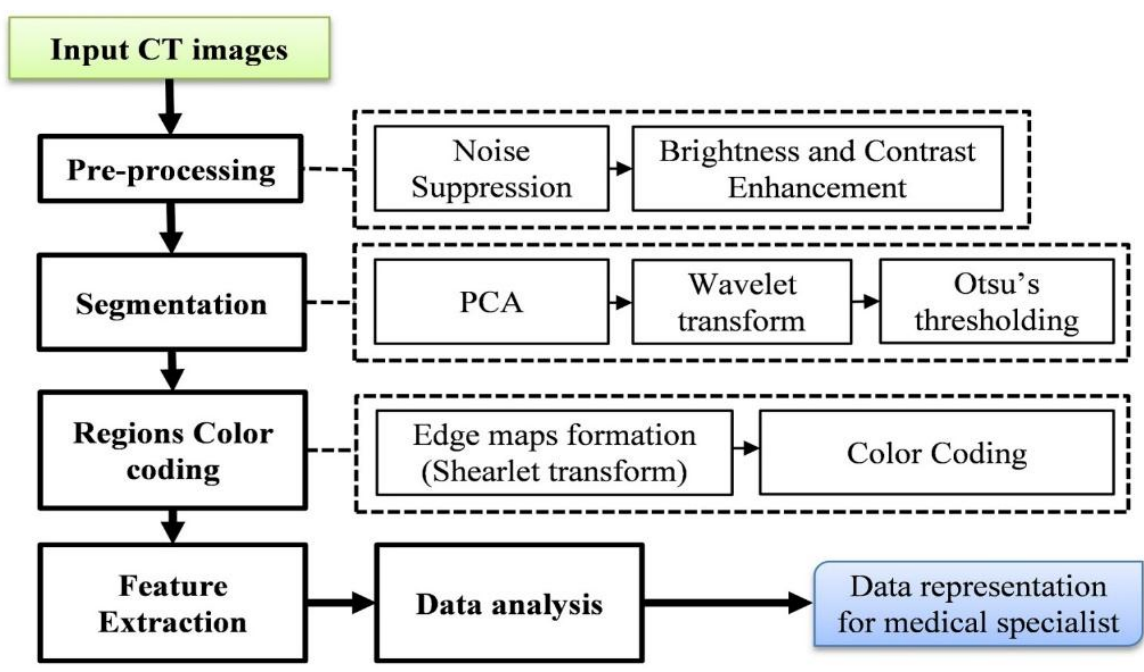

Figure 1. Flow-chart of image processing.

\section{METHODS}

The main stages of image research are the selection of the area of interest, segmentation and extraction of the main markers (Fig. 1). Segmentation and visualization of pathological changes on the studied CT images was carried out for further processing and texture analysis of images in order to solve diagnostic problems in dynamics. The use of methods for preliminary processing of CT images can significantly improve the quality of the selection of the contours of objects of interest. The modified segmentation algorithm for identification of the pathological areas of the lungs and local objects of interest is quite effective in terms of accuracy and speed. Thus, within the framework of computational technology it is proposed to use algorithms for noise reduction, contrast enhancement, segmentation and spectral decomposition (Shearlet transform).

The main task of pre-processing is to improve the quality of CT images. This step allows to improve certain image parameters (for example, the signal-to-noise ratio), and improve the visual representation by removing noise, as well as, modifying unwanted parts of the background. As a preliminary processing for noise reduction, it was decided to use a median filter with modifications for image set processing. Also it was decided to use the weights taking into account the distance of adjacent CT images from the processed image. The implementation of the median filter based on the histograms (Zotin et al., 2020) is taken as the basis. Improvement of the quality of noise reduction is based on adaptive weight adjustment based on local neighborhood $(3 \times 3)$ of the processed images. The scheme of the histogram formation taking into account the weight is shown in Fig. 2.

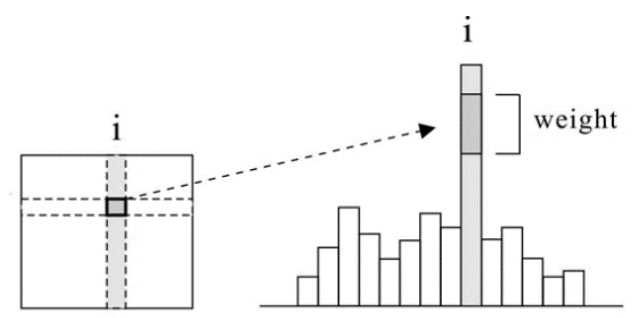

Figure 2. Scheme of weighted median filter histogram formation.
Since CT images can have different brightness and contrast, it was decided to use the Multi-Scale Retinex (MSR) modification, in which wavelet transform was used to speed up the calculations (Green, O. 2018) to correct brightness and local contrast in the area of interest. A brighter representation in the lung area allows to calculate texture features and to assess the structural characteristics of the lesion.

Therefore, based on models of the texture (geometric) analysis, local objects of interest are selected and contrasted taking into account the use of color coding (Zotin et al., 2018). The essence of the study is the analysis of the texture (homogeneity and entropy) of the zone of pathological changes with the adjustment of the intensity level and its adaptation in the analysis of histograms.

Segmentation methods are applicable to solving various problems in the analysis of pathological changes. For example, in the image below (Fig. 3), in the presence of an infected parenchyma in both lungs, the affected areas were segmented.

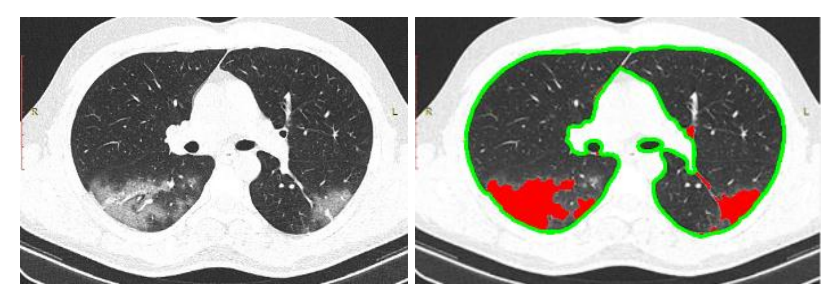

Figure 3. Segmentation of areas of the parenchyma involved.

Further, with the help of Shearlet transformation various color variations are formed as shown in Fig. 4. More and less dense zones in the area of the infected parenchyma (red and yellow colors) are highlighted in relation to the air tissue of the lung (blue). 

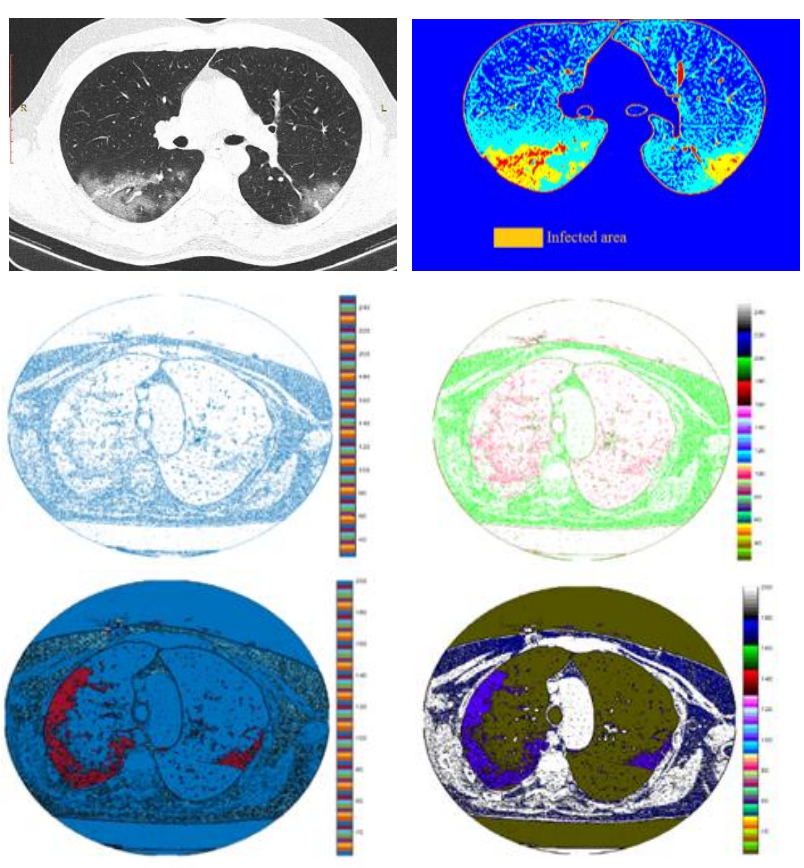

Figure 4. Examples of segmentation and color coding of lungs with COVID-19.

In the series above the presented images the zones associated with COVID-19 are highlighted the most representative image with zones of red and purple colors in relation to the blue or green color of the unchanged pulmonary parenchyma. Also interesting is the moment of the ratio of soft tissues and cellulose, also of different colors, which is associated with the different density of these structures.

\section{EXPERIMENTS AND RESULTS}

In the experimental part of the study, using a database of computed tomography, the results of calculations were obtained to assess lung pathologies associated with COVID-19 (Handbook of COVID-19 Prevention and Treatment, 2020). A series of calculations was carried out to visualize the affected areas from two-dimensional sections (patterns) of computed tomography of the lungs. The computational procedure for calculating the degree of lung damage makes it possible to determine the boundaries of the pathology zone with increased accuracy. This, in turn, leads to an increase in the accuracy of the area estimate and the assessment of the severity of the disease.

As part of an experimental study a CT image database was formed to assess lung pathologies associated with COVID-19 (Zotin et al., 2020). When forming the database the selection of images occurs only in verified patients divided into groups according to the severity of the disease (CT1-CT4) with dynamic images. This makes it possible to track changes in the affected area over time (Li and Xia, 2020).

The database is formed from patients with a confirmed virus and divided into groups according to severity (CT1-CT4) and a set of images taken over time. For a visual demonstration, examples of two patients with different severity and different ages are considered below.
When analyzing CT scans images of two different patients with confirmed coronavirus infection were used (men: 24 years old patient 1 and 73 years old - patient 2). The first patient has CT1 severity $(<25 \%$ of the affected parenchyma), the second patient has CT3 (> 50\% of the affected parenchyma). These patients underwent a retrospective image analysis with signs of COVID19.

\subsection{Analysis of the CT images of the first patient}

The first patient is 24 years old with mild severity and no concomitant pathology. Was admitted to the hospital with a complaint only about an increase in temperature and the presence of a positive smear. During CT scans double-sided areas of the "frosted glass" type were noted (Fig. 5).

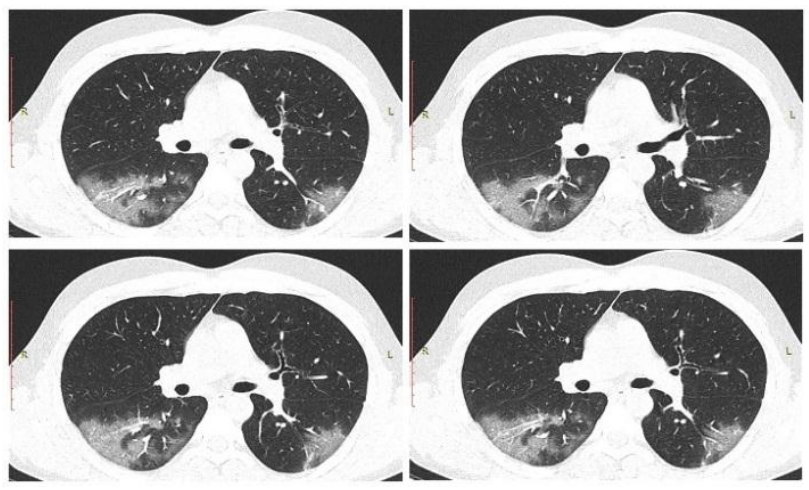

Figure 5. CT CT image of patient 1, first study.

In these pictures mainly the lower lobes of both lungs are affected. Dynamic observation showed a correlation with the stage of the disease. At the early stage of observation (1-4 days), the main textural indicator is the "ground-glass". At the next stage of progression (5-8 days) the appearance of foci of compaction and an indicator of the "cobblestone" type is noted. The peak stage (9-13 days) is characterized by the predominance of the "consolidation" type. At the stage of resolution (> 14 days) - there is a regression of the identified changes. These stages can differ in temporal and structural characteristics depending on a number of criteria and dynamics of the process. During dynamic observation in this patient in the stage of progression there is a consolidation of these changes (Fig. 6).

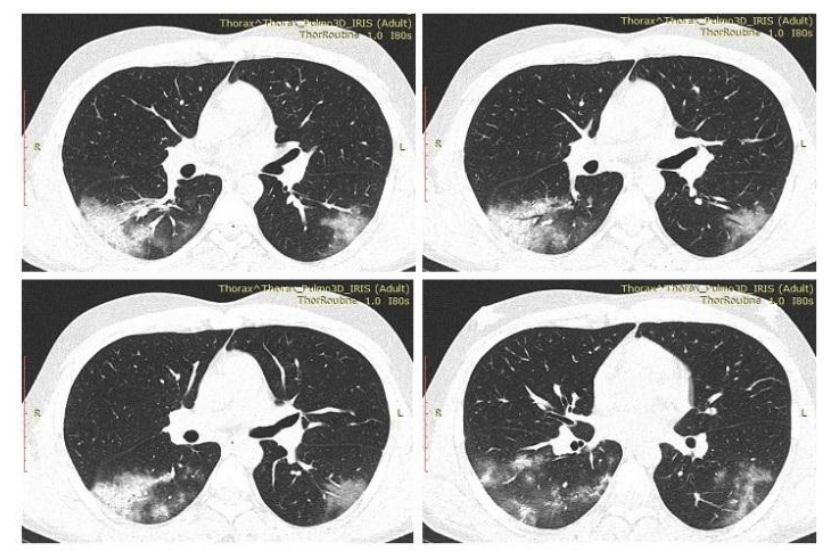

Figure 6. CT image of patient 1, follow-up, progression stage. 
In a retrospective analysis it was shown that given the small extent of the lesion and young age as well as with the appropriate therapy a quick recovery was observed which is shown in Fig. 7.

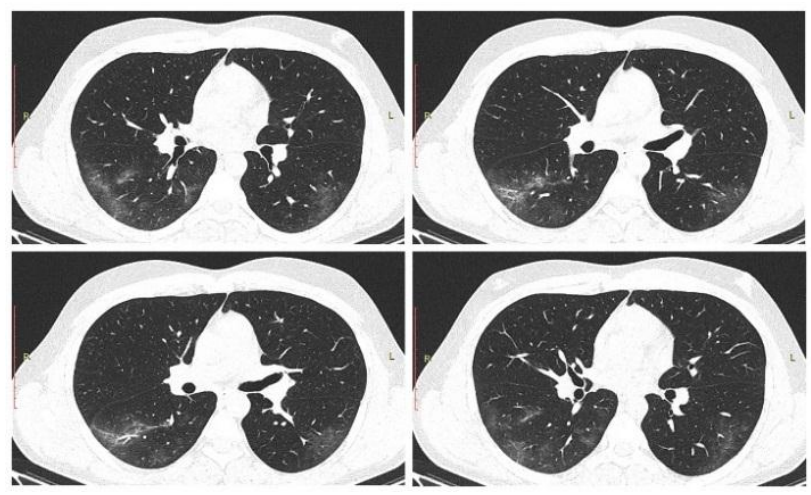

Figure 7. CT image of patient 1, follow-up, resolution stage.

Using the computational technology developed by the authors the parenchyma involved was assessed with differentiation and tracking of zones by shear density characteristics presented in a series of images (Fig. 8). So for each image the results of segmentation, selection of the pathology area and definition are presented: the first column of images shows the boundaries of change. The second column of the images shows the selection and estimation of the area of changes. Further, the subsequent division into more and less dense areas is performed which is shown in the images in the third column.

a

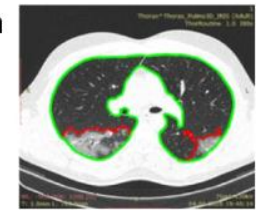

b
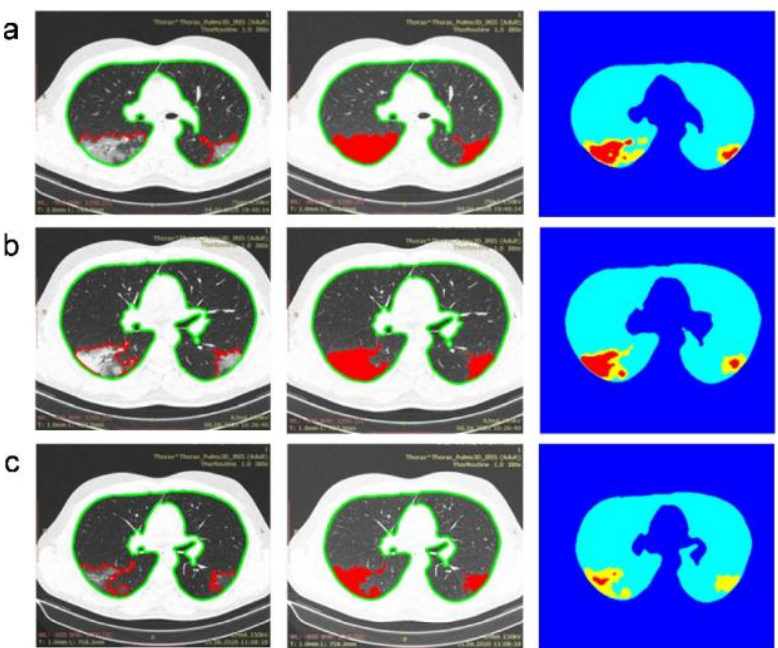

Figure 8. Examples of CT image processing of lungs with COVID-19 (1st patient): a) observation on the 3rd day of illness; b) observation on the 7th day of illness; c) observation on the 14th day of illness.

Positive regression in patient 1 a small percentage of denser changes and their positive dynamics one can assume the patient's exit from long-term changes in the lungs.

\subsection{Analysis of the CT images of the second patient}

The second patient, who is used to demonstrate the proven technique, is an older patient (73 years old). He had a greater percentage of involved lung parenchyma and had complaints of fever, chest pain and cough. The CT scan was also performed and more extensive areas of damage were identified (Fig. 9).

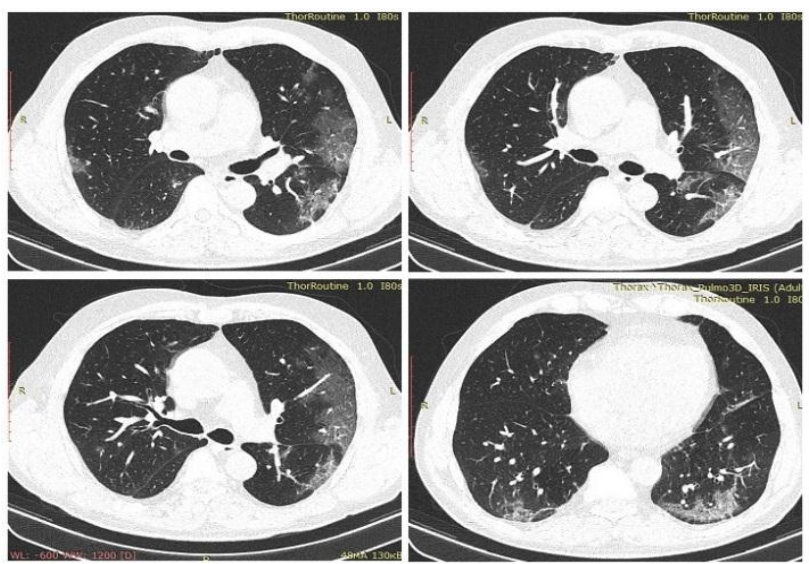

Figure 9. CT image of patient 2, first day of illness.

A dynamic study reveals the compaction of the revealed changes with the formation of tense zones, which is shown in a series of images (Fig. 10).

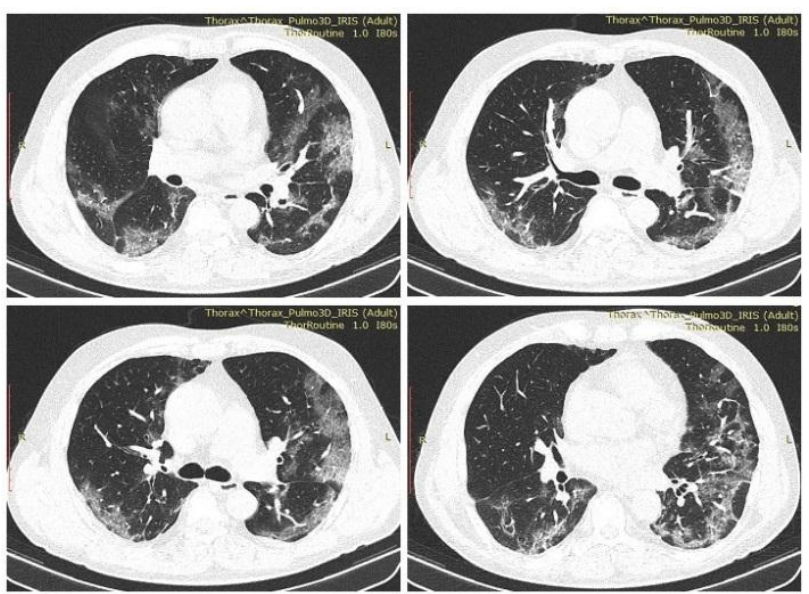

Figure 10. CT images of patient 2, dynamics -7 days.

On day 14, the CT scan of the lungs was also performed in which the changes persisted, but with a more pronounced reticular component (Fig. 11).

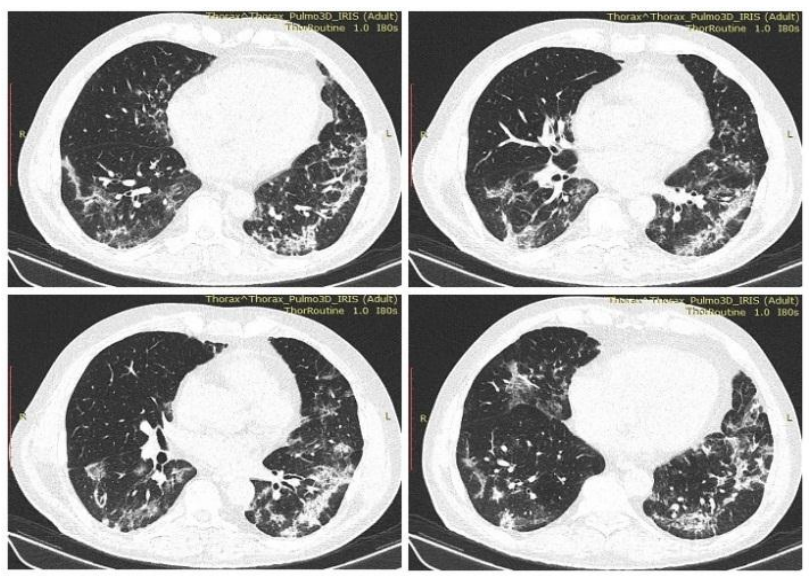

Figure 11. CT images: signs of gross reticular changes. 
When applying the computational technique for the analysis of CT images of the second patient, the area of the lesion in the lungs was also calculated and their density changes were assessed (Fig. 12).

a

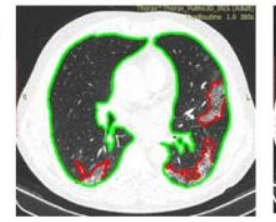

b

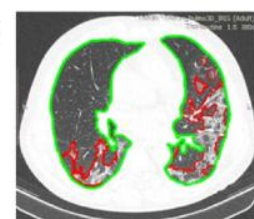

C

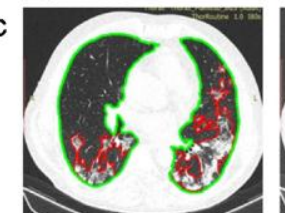

Figure 12. Examples of CT image processing of lungs with COVID-19 (2nd patient): a) observation on the 3rd day of illness; b) observation on the 7 th day of illness; c) observation on the 14th day of illness.

When the second patient was discharged, the revealed changes remained in his lungs, requiring dynamic observation. In retrospective analysis it should be assumed that the patient may have long-term consequences and residual changes (in the area of denser red zones).

When carrying out dynamic studies in the second patient a pronounced positive trend can be observed. The coarse zones of reticular changes disappeared with the preservation of residual zones of low intensity like "ground-glass" and insignificant reticular changes (Fig. 13).
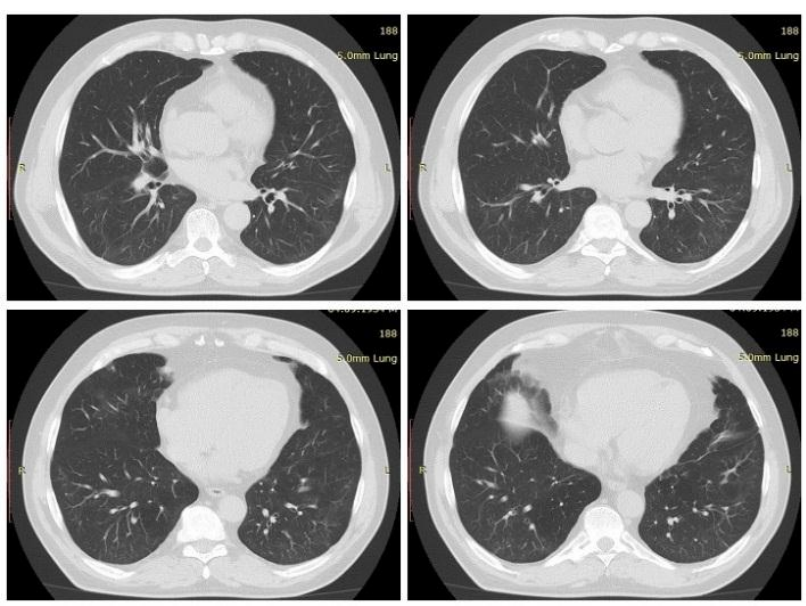

Figure 13. CT images of the $2^{\text {nd }}$ patient, follow-up after 6 months.

If the outcome is favorable, as in the case of this patient, only minor residual changes are observed.

\section{DISCUSSION}

In retrospective analysis, it should be assumed that patient 2 may have long-term consequences and residual changes (in the area of denser red zones) (Kents et al., 2020). When carrying out dynamic studies in the second patient a pronounced positive dynamics is observed with the disappearance of coarse zones of reticular changes. At the same time, there was a preservation of the residual zones of low intensity such as "opaque haze".

We observe a favorable outcome, since in this case only minor residual changes remain. In the dynamic analysis of this patient taking into account changes in the lungs he was initially included in the group of patients with possible preservation of residual effects and the formation of fibrosis. In this case the formation of fibrosis is not observed. The results obtained by experts were almost equal to computer processing with a slight deviation for a number of points which was taken as a standard.

Comparison of the lesion volume for two patients is shown in the summary table with a number of other indicators on the day of discharge (Table 1). The volume of the lesion was determined averaged over the most representative sections in the sum for both lungs. In this case, this is not an indicator of the degree of severity, but only marks the correlation of changes from the stage of the disease.

\begin{tabular}{|l|c|c|}
\hline \multicolumn{1}{|c|}{ Evaluated Features } & $\begin{array}{c}\text { First patient } \\
\text { (CT1) }\end{array}$ & $\begin{array}{c}\text { Second patient } \\
\text { (CT3) }\end{array}$ \\
\hline Bilateral Lungs Involvement & + & + \\
Volume: & $14 \%$ & $12 \%$ \\
First CT & $12 \%$ & $28 \%$ \\
Day 7 & $9 \%$ & $26 \%$ \\
Last CT & Unique & Multiple \\
GGO (ground glass opacity) & - & + \\
Reticular changes
\end{tabular}

Table 1. The location, morphology and volume of pulmonary lesions in different types in Coronavirus Disease-19 patients.

In the second patient the preservation of the lesion volume on the last CT images should be noted. On visual and clinical analysis, these manifestations changed the structure in the lungs without the presence of complaints and clinical components. In this case, we can talk about their longer regression, which was confirmed in the study (Table 2).

\begin{tabular}{|l|c|c|}
\hline Evaluated Features & $\begin{array}{c}\text { Evaluation by } \\
\text { radiologist, \% }\end{array}$ & $\begin{array}{c}\text { Software } \\
\text { evaluation, \% }\end{array}$ \\
\hline Pathology localization & $94.1 \pm 3.2$ & $97.7 \pm 4.8$ \\
Volume & $92 \pm 3.1$ & $98.1 \pm 5$ \\
Pathology objects detection & $93.3 \pm 5.1$ & $98.9 \pm 3.4$ \\
Shape & $96.2 \pm 1.9$ & $99.5 \pm 2.1$ \\
Size of pathologies' structural & 0.0 & $99.3 \pm 1.5$ \\
elements & $92.1 \pm 2.0$ & $98.5 \pm 1.8$ \\
Edge & 100.0 & 0.0 \\
Relation to other structures & $90.7 \pm 4.9$ & $98.3 \pm 1.6$ \\
Tissue density & $98.2 \pm 0.8$ & 100.0 \\
Sensitivity &
\end{tabular}

Table 1. Assessment of the definition of indicators and volume, performed by medical specialists manually and using the proposed representations generated by software. 


\section{CONCLUSIONS}

We have shown the possibility to obtain additional information about the parameters of the texture (markers) which with subsequent interpretation makes it possible to expand the $\mathrm{X}$-ray diagnostic opinion of the radiologist (Rodriguez-Morales et al., 2020). A quantitative assessment of the degree of involvement of the lung parenchyma in the pathological process (in percentage) was carried out.

The possibility of dynamic observation with the formation of a forecast of the patient's condition is shown. The criteria derived from experimental studies can be useful indicators in predicting treatment outcomes. This is due to the fact that long-term preservation of the dense zone may indicate the manifestation of fibrosis. Thus, if during the period of dynamic observation this pathology remains unchanged both visually and structurally in the same volume then the likelihood of fibrosis in this area increases.

\section{REFERENCES}

Bernheim, A., Mei, X., Huang, M., Yang, Y., Fayad, Z.A., Zhang, N., Diao, K., Lin, B, Zhu, X., Li, K., Li, S., Shan, S., Jacobi, A., Chung, M., 2020: Chest CT findings in coronavirus disease-19 (COVID-19): relationship to duration of infection. Radiology 295 (3). https://doi.org/10.1148/radiol.2020200463

Green, O., 2018: Efficient scalable median filtering using histogram-based operations. IEEE Trans-actions on Image Processing 27 (5), 2217-2228.

DOI: 10.1109/TIP.2017.2781375

Handbook of COVID-19 Prevention and Treatment. 2020. In: Editor, Liang, T., edn. Zhejiang University School of Medicine.

Kents A., Hamad Y., Simonov K., Zotin A., Kurako M., 2020: Geometric analysis of pathological changes in the lungs using CT images for COVID-19 diagnosis. Short Paper Proceedings of the 1st Siberian Scientific Workshop on Data Analysis Technologies with Applications, CEUR Workshop Proceedings (ISSN 1613-0073), 2727, 43-50.

Li, Y., Xia, L., 2020: Coronavirus disease 2019 (COVID-19): Role of Chest CT in diagnosis and management. American Journal of Roentgenology 214 (6), 1280-1286.

doi: 10.2214/AJR.20.22954

Liu, K.C., Xu, P., Lv, W.F., Qiu, X.H., Yao, J.L., Gu, J.F., Wei, W., 2020: CT manifestations of coronavirus disease-2019: A retrospective analysis of 73 cases by disease severity. Eur. J. Radiol. 126, 108941. doi: 10.1016/j.ejrad.2020.108941

Long, J.B., Ehrenfeld, J.M., 2020: The role of augmented intelligence (AI) in detecting and preventing the spread of novel coronavirus. J. Med Syst. 44(3), 59. doi: 10.1007/s10916-0201536-6

McDonald, R.J., Schwartz, K.M., Eckel, L.J., Diehn, F.E., Hunt, C.H., Bartholmai, B.J., Erickson, B.J., Kallmes D.F., 2015: The effects of changes in utilization and technological advancements of cross-sectional imaging on radiologist workload. Acad. Radiol. 22(9), 1191-1198.

doi: 10.1016/j.acra.2015.05.007. Epub 2015 Jul 22.
Radiation diagnosis of coronavirus disease (Covid-19): organization, methodology, interpretation of results, 2020. Preprint No. CDT - 2020 - II, Version 2 (17.04.2020).

Shen, C., Yu, N., Cai, S., Zhou, J., Sheng, J., Liu, K., Zhou, H., Guoa, Y., Niu, G., 2020: Quantitative computed tomography analysis for stratifying the severity of Coronavirus Disease. Journal of Pharmaceutical Analysis 10(2), 123-129. https://doi.org/10.1016/j.jpha.2020.03.004

Yasar, H., Ceylan, M., 2021: A novel comparative study for detection of Covid-19 on CT lung images using texture analysis, machine learning, and deep learning methods. Multimed Tools Appl. 80, 5423-5447. https://doi.org/10.1007/s11042-020-09894-3

Zotin, A., Hamad, Y., Simonov, K., Kurako, M., Kents, A., 2020: Processing of CT Lung Images as a Part of Radiomics. In: Czarnowski, I., Howlett, R., Jain, L. (eds). Intelligent Decision Technologies. IDT 2020. Smart Innovation, Systems and Technologies 193, 243-252. Springer, Singapore.

Zotin, A., Simonov, K., Kapsargin, F., Cherepanova, T., Kruglyakov, A. 2020: Tissue germination evaluation on implants based on shearlet transform and color coding. In: Favorskaya, M.N., Jain, L.C. (eds) Computer Vision in Advanced Control Systems-5. ISRL, 175, 265-294. Springer, Cham. 